\title{
Chromosome Identification and Characterization in Trifoliate Orange (Poncirus trifoliata (L.) Raf.) by CMA and PI/DAPI Staining and GISH
}

\author{
Bannarat Preedasuttijit $^{1}$, Akira Kitajima ${ }^{2 *}$, Atsu Yamasaki ${ }^{2}$, Tsuneo Ogata ${ }^{3}$ \\ and Kojiro Hasegawa ${ }^{3}$ \\ ${ }^{1}$ The United Graduate School of Agricultural Science, Ehime University, Tarumi, Matsuyama 790-8566, Japan \\ ${ }^{2}$ Graduate School of Agriculture, Kyoto University, Hattyonawate, Takatsuki 569-0096, Japan \\ ${ }^{3}$ Faculty of Agriculture, Kochi University, Monobe, Nankoku 783-0093, Japan
}

The chromosomes of trifoliate orange (Poncirus trifoliata (L.) Raf.) were stained with chromomycin $A_{3}$ (CMA) and double stained with alkylating fluorochromes 4'-6-diamidino-2-phenylindole (DAPI) and propidium iodide (PI). PI-positive (PI (+)) regions in PI/DAPI staining were identical to CMA-positive (CMA (+)) regions. The signals of PI $(+)$ were more stable than those of CMA (+). Based on the relative-sized PI $(+)$ signals and the morphology of no-signal chromosomes, eighteen chromosomes of trifoliate orange were classified into 9 groups. Genomic in situ hybridization (GISH) was performed on trifoliate orange chromosomes using the double probe of DNA from trifoliate orange labeled with digoxigenin-rhodamine (red), and from 'Nankan No. 20' satsuma mandarin (Citrus unshiu Marcow.) or 'Tosa Buntan' pummelo (C. maxima (Burm.) Merr.) labeled with biotinFITC (green). Sixteen GISH signals with a higher intensity were detected in the identical positions of PI (+) regions. The coloration of GISH showed 13 yellow signals and 1 green signal by the biotin probes of satsuma mandarin, and 5 yellow signals and 11 red signals by those of 'Tosa Buntan' pummelo. This indicates that trifoliate orange is more closely related to satsuma mandarin than 'Tosa Buntan' pummelo, because red signals indicate that they share no homologous sequences between genomes. GISH signals on the secondary constriction regions of all B-type chromosomes and on the telomeric region of the $D_{4}$-type chromosome were yellow or green in both satsuma mandarin and 'Tosa Buntan' pummelo biotin probes. This result suggests that these regions in trifoliate orange chromosomes can be homologous to satsuma mandarin and 'Tosa Buntan' pummelo genomes, and are thus conserved regions.

Key Words: chromosome, citrus, GISH, PI/DAPI, trifoliate orange.

\section{Introduction}

Within Citrus and its closely related genera, Poncirus and Fortunella, high cross-compatibility has led to natural interspecific and intergeneric hybrids, resulting in numerous morphological variations. Because of their heterozygosity, the taxonomic systems among the citrus group are still controversial. Recently, studies on classification in Citrus and its related genera (Coletta Filho et al., 1998; Fang et al., 1998; Yamamoto and Kobayashi, 1996) have been conducted based on DNA analysis which indicates that citron, pummelo, and mandarin are basic species in the genus Citrus.

Received; June 19, 2006. Accepted; January 11, 2007.

* Corresponding author (E-mail: kitajima@kais.kyoto-u.ac.jp).
Furthermore, chromosome research involving taxonomy and the genome has been conducted in Citrus and its closely related genera (Befu et al., 2000; Miranda et al., 1997a). In addition to the taxonomic and genomic impacts of chromosome research, it could be important to citrus breeding as well. For example, it has been reported that resistance to the citrus tristeza virus (CTV), which is a major viral pathogen of citrus, is controlled by a single dominant gene called Ctv located on a trifoliate orange (Poncirus trifoliata (L.) Raf.) chromosome (Gmitter et al., 1996; Yoshida, 1985). Therefore, a better understanding of chromosome identification in trifoliate orange will help in the development of corresponding physical map for $C t v$.

The discrimination of citrus chromosomes during somatic metaphase is difficult because of their small sizes $(1-3.5 \mu \mathrm{m})$ and similar morphology (Befu et al., 
2000). The chromosome number, $2 \mathrm{n}=18$, is constant in citrus, with the exception of some cultivated polyploids (Cameron and Frost, 1968; Krug, 1943). Some authors have also analyzed citrus karyotypes based on Giemsa C-banding (Guerra, 1985; Liang, 1988; Wei et al., 1988) and staining with intercalating fluorochromes of chromomycin $\mathrm{A}_{3}$ (CMA) and 4'-6-diamidino-2phenylindole (DAPI) (Guerra, 1993), and showed that many chromosome pairs must be heteromorphic, perhaps as a consequence of widespread interspecific hybridization during the evolution of the major domesticated taxonomy. CMA band patterns of citrus chromosomes can be classified into at least 5 types (Befu et al., 2001; Miranda et al., 1997a), that is, A-type with three CMApositive signals on both of the two telomeric regions and secondary constriction region, B-type with two positive signals on the telomeric and secondary constriction regions, C-type with two positive signals on both telomeric regions, D-type with one positive signal on the telomeric region, and E-type with no positive signals. Befu et al. (2001) demonstrated that D-type and E-type would be basic types in Citrus. The potential CMA staining method could be an excellent tool for karyotyping. Although trifoliate orange chromosomes were grouped into 3 categories based on the CMA banding pattern (4B $+8 \mathrm{D}+6 \mathrm{E})$ by Befu et al. (2000), a more detailed chromosome identification is necessary for physical mapping.

Genomic in situ hybridization (GISH), using genomic probes from parental plants or putative progenitor species, has been used to identify parental chromosomes, alien chromatin in interspecific hybrids, and genomic compositions in many plant species. GISH successfully identified progenitor species in horticultural plants such as Allium wakegi (Hizume, 1994) and Coffea arabica (Raina et al., 1998). Furthermore, GISH has been used to investigate the genomic composition and phylogenetic relationships in Diospyros species (Choi et al., 2003). Thus, GISH signals may provide more information on genomic composition and identification of each chromosome in trifoliate orange.

In this study, chromosome identification of trifoliate orange was conducted at the prometaphase stage of chromosomes by CMA and PI/DAPI fluorochrome staining and the GISH method to investigate the genomic composition and phylogenetic relationships between trifoliate orange, 'Nankan No. 20' satsuma mandarin (C. unshiu Marcow.), and 'Tosa Buntan' pummelo (C. maxima (Burm.) Merr.).

\section{Materials and Methods}

\section{Plant materials and chromosome preparation}

The chromosome preparation method was followed that of Kitajima et al. (2001). Young spring leaves, about $3-5 \mathrm{~mm}$ long, from trifoliate orange were immersed in $2 \mathrm{mM}$ 8-hydroxyquinoline for $3 \mathrm{~h}$ at $20^{\circ} \mathrm{C}$ and fixed in methanol-acetic acid $(3: 1, v / v)$. Fixed leaves were finely cut after washing in water and digested with an enzyme solution of $0.3 \%(\mathrm{w} / \mathrm{v})$ Cellulase Onozuka RS (Yakult, Japan) and $0.2 \%(w / v)$ Pectolyase Y-23 (Kikkoman, Japan) in $75 \mathrm{mM} \mathrm{KCl}$ for $3 \mathrm{~h}$ at $37^{\circ} \mathrm{C}$. Then, a ten-times volume of a $\mathrm{KCl} 75 \mathrm{mM}$ solution for the enzyme solution was added and incubated for $1 \mathrm{~h}$ at $37^{\circ} \mathrm{C}$. The digested samples were centrifuged at $1500 \mathrm{rpm}$ for $5 \mathrm{~min}$ and washed twice with the fixative solution. After the final rinsing, $60 \mu \mathrm{L}$ of residue was suspended and dropped from a height of $30 \mathrm{~cm}$ for the critical efficiency. After overnight air-drying, the slide was stained with a $2 \%$ Giemsa (Sigma-Aldrich Japan, Japan) solution for 15 min. Good-spread chromosomes were checked by a microscope (BX-50, OLYMPUS, Japan) and photographed with a camera system (PM-20, OLYMPUS, Fuji Color ISO 100, Fuji Film, Japan).

\section{CMA staining}

The preparations with good-spread chromosomes were destained by immersion in an ethanol series of $70 \%, 90 \%$, and $100 \%$ for 10,5 , and $5 \mathrm{~min}$, respectively. The dried slides were treated with McIlvaine's buffer (pH 7.0) for $15 \mathrm{~min}, 0.2 \mathrm{~g} \cdot \mathrm{L}^{-1}$ of distamycin A for $10 \mathrm{~min}$, $5.0 \mathrm{mM}$ of a $\mathrm{MgCl}_{2}$ buffer solution for $10 \mathrm{~min}$, and $50 \mu \mathrm{L}$ of $0.12 \mathrm{~g} \cdot \mathrm{L}^{-1}$ chromomycin $\mathrm{A}_{3}$ (CMA) for $1 \mathrm{~h}$ at room temperature under dark conditions, and then rinsed in McIlvaine's buffer for $10 \mathrm{~min}$. The preparations were counterstained with $50 \mu \mathrm{L}$ of $1 \mathrm{~g} \cdot \mathrm{L}^{-1} 4^{\prime}$-6-diamidino-2phenylindole (DAPI) and mounted in glycerol 1:1 (v/v). The chromosomes were observed with an epifluorescence microscope (BX-FLA, OLYMPUS) equipped with a BV filter and photographed by the camera system.

\section{PI/DAPI staining}

The destained preparations were counterstained with $50 \mu \mathrm{L}$ of propidium iodide (PI) consisting of $10 \%(\mathrm{v} / \mathrm{v})$ $1 \times$ PBS $(-), 2 \%(\mathrm{w} / \mathrm{v})$ 1,4-diazabicyclo[2,2,2]-octane (DABCO), $1 \mathrm{~g} \cdot \mathrm{L}^{-1} \mathrm{PI}$, and $50 \mu \mathrm{L}$ of $1 \mathrm{~g} \cdot \mathrm{L}^{-1}$ DAPI for $2 \mathrm{~h}$ at $4^{\circ} \mathrm{C}$. The chromosomes were observed with an epifluorescence microscope equipped with a WU filter and photographed by the camera system.

\section{Probe preparation for GISH}

Total genomic DNA was isolated from $1.5 \mathrm{~g}$ of immature spring leaves of 'Nankan No. 20' satsuma mandarin, 'Tosa Buntan' pummelo, and trifoliate orange by the CTAB method (Doyle and Doyle, 1987) and adjusted to $200 \mathrm{mg} \cdot \mathrm{L}^{-1}$ in concentration. The adjusted DNA was labeled by a Nick translation kit according to the manufacturer's protocol (La Roche Ltd., Switzerland) with digoxigenin-11-dUTP (Dig) in trifoliate orange, and with biotin-16-dUTP (Bio) in satsuma mandarin and 'Tosa Buntan' pummelo. Each labeled DNA was resolved in $20 \mu \mathrm{L}$ formamide (probe solution).

\section{GISH}

Dig-labeled DNA of trifoliate orange was combined 
with Bio-labeled DNA of satsuma mandarin or 'Tosa Buntan' pummelo as a double probe. Ten $\mu \mathrm{L}$ of a Diglabeled trifoliate orange probe solution and $10 \mu \mathrm{L}$ of a Bio-labeled probe solution were mixed (probe mixture). The probe mixture was denatured at $80^{\circ} \mathrm{C}$ for $10 \mathrm{~min}$, cooled rapidly on ice for at least $10 \mathrm{~min}$, and then $15 \mu \mathrm{L}$ of $20 \%$ dextran sulfate was added to it in $4 \times$ SSC.

Adequate preparations were denatured in $2 \times \mathrm{SSC}$ with $70 \%$ formamide at $70^{\circ} \mathrm{C}$ for $5 \mathrm{~min}$ after aging in $4 \times \mathrm{SSC}$ with $0.1 \%$ triton $\mathrm{X}$ at $37^{\circ} \mathrm{C}$ for $30 \mathrm{~min}$ and dehydration. The denatured probe mixture was dropped on the preparations and hybridized overnight in a humid chamber at $37^{\circ} \mathrm{C}$. Post-hybridization washing was done with $50 \%$ formamide in $2 \times \mathrm{SSC}$ at $37^{\circ} \mathrm{C}$, and $2 \times \mathrm{SSC}$, $1 \times \mathrm{SSC}$, and $4 \times \mathrm{SSC}$ at room temperature for $15 \mathrm{~min}$. Hybridization sites were detected using anti-Digrhodamine (red color) and avidin-fluorescein isothiocyanate (green color). GISH preparations were counterstained with DAPI and mounted in a $1 \%(w / v) p$ phenylene diamine (PPD) antifade solution. GISH chromosomes were photographed with an epifluorescence microscope (BX-FLA) and a camera system (PM20, Fuji Color ISO 100) by double exposure with IB and IG filters.

\section{Results}

\section{CMA and PI/DAPI staining}

Chromosomes of trifoliate orange stained with CMA and PI/DAPI are shown in Figure 1. CMA and PI/DAPI staining made it possible to observe the banding patterns of trifoliate orange chromosomes, according to the positions of CMA-positive (CMA (+)) and PI-positive (PI (+)) regions. PI/DAPI staining led to bright pink signals of PI $(+)$ regions, while CMA staining gave yellow signals. PI $(+)$ regions were completely identical to CMA (+) regions, and the total number of positive signals was 16 , distributed in 12 out of 18 chromosomes. PI (+) signals were brighter and more stable than CMA $(+)$ signals. Based on the banding patterns of positive signals following Miranda et al. (1997a) and Befu et al.
(2000), trifoliate orange chromosomes were classified into 3 types, that is, a B-type which contained two positive signals, one on the telomeric region and the other on the secondary constriction, D-type which contained one positive signal on the telomeric region, and E-type which had no positive signals. The trifoliate orange chromosomes based on PI (+) patterns were classified into $4 \mathrm{~B}+8 \mathrm{D}+6 \mathrm{E}$, which was the same as the result based on CMA $(+)$ patterns.

Chromosomes with the same types of banding patterns could be classified by the relative size of PI (+) regions and morphological characteristics. Four chromosomes of the B-type were grouped into 3 categories $\left(\mathrm{B}_{1}, \mathrm{~B}_{2}\right.$, and $\mathrm{B}_{3}$ ) based on combinations of the relative size of 2 PI (+) signals (Figs. 2 and 3). In the $\mathrm{B}_{1}$-type, two PI (+) signals were represented at a relatively medium size on both the secondary constriction and the telomeric region. The PI (+) regions on the secondary constriction were often separate from each other because of secondary constriction stretching. In the $\mathrm{B}_{2}$-type, one signal was small on the secondary constriction and the other signal was large on the telomeric region. In the $\mathrm{B}_{3}$-type, one was large on the secondary constriction and the other was small on the telomeric region. D-type chromosomes were also grouped into 4 categories $\left(D_{1}, D_{2}, D_{3}\right.$, and $\mathrm{D}_{4}$ ) by the relative size of PI (+) signals, that is, $\mathrm{D}_{1-}$ type with an intermediate signal size, $\mathrm{D}_{2}$-type with large signals, $\mathrm{D}_{3}$-type with small signals, and $\mathrm{D}_{4}$-type with small and long signals on the sharp morphology of the telomeric region. E-type chromosomes which had no PI $(+)$ region were categorized into $\mathrm{E}_{\mathrm{L}}$ (relatively long with a distinct centromere) and $\mathrm{E}$ (other E-type chromosomes). All 18 chromosomes of trifoliate orange were classified by PI (+) band patterns with relative sizes of their signals and morphological characteristics into two type- $\mathrm{B}_{1}$, one type- $\mathrm{B}_{2}$, one type- $\mathrm{B}_{3}$, four type- $\mathrm{D}_{1}$, two type- $\mathrm{D}_{2}$, one type- $\mathrm{D}_{3}$, one type- $\mathrm{D}_{4}$, two type- $\mathrm{E}_{\mathrm{L}}$, and four type- $\mathrm{E}\left(2 \mathrm{~B}_{1}+1 \mathrm{~B}_{2}+1 \mathrm{~B}_{3}+4 \mathrm{D}_{1}+2 \mathrm{D}_{2}+1 \mathrm{D}_{3}+1 \mathrm{D}_{4}+\right.$ $2 \mathrm{E}_{\mathrm{L}}+4 \mathrm{E}$ ), as demonstrated in Figure 3 .

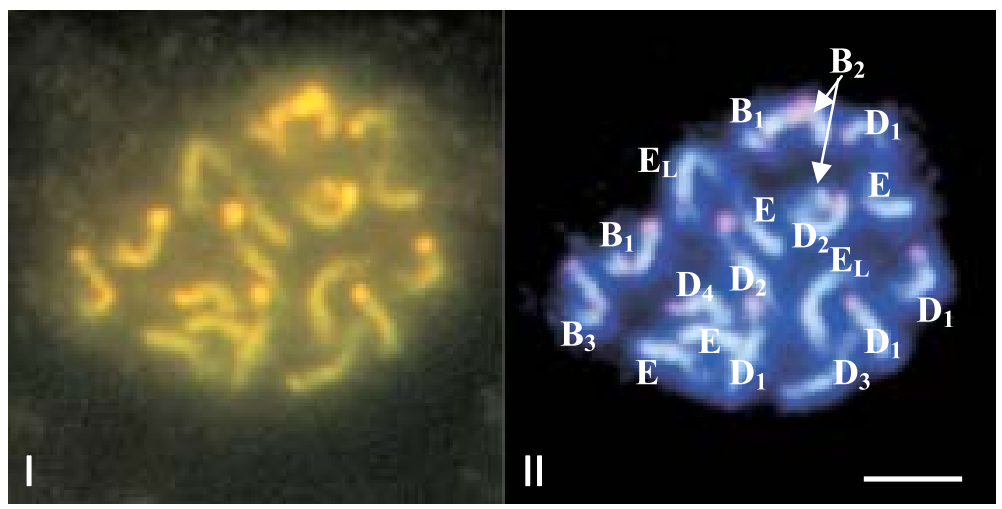

Fig. 1. Chromosomes of trifoliate orange stained with chromomycin $\mathrm{A}_{3}$ (I) and propidium iodide (PI)/4'-6-diamidino-2-phenylindole (DAPI) (II). The letters $\mathrm{B}_{1}, \mathrm{~B}_{2}, \mathrm{~B}_{3}, \mathrm{D}_{1}, \mathrm{D}_{2}, \mathrm{D}_{3}, \mathrm{D}_{4}, \mathrm{E}_{\mathrm{L}}$, and $\mathrm{E}$ in photograph II indicate chromosome types, respectively, $2 \mathrm{n}=18$. Arrows indicate one $\mathrm{B}_{2}$-type chromosome which contains a stretched segment. Scale bar indicates $5 \mu \mathrm{m}$. 


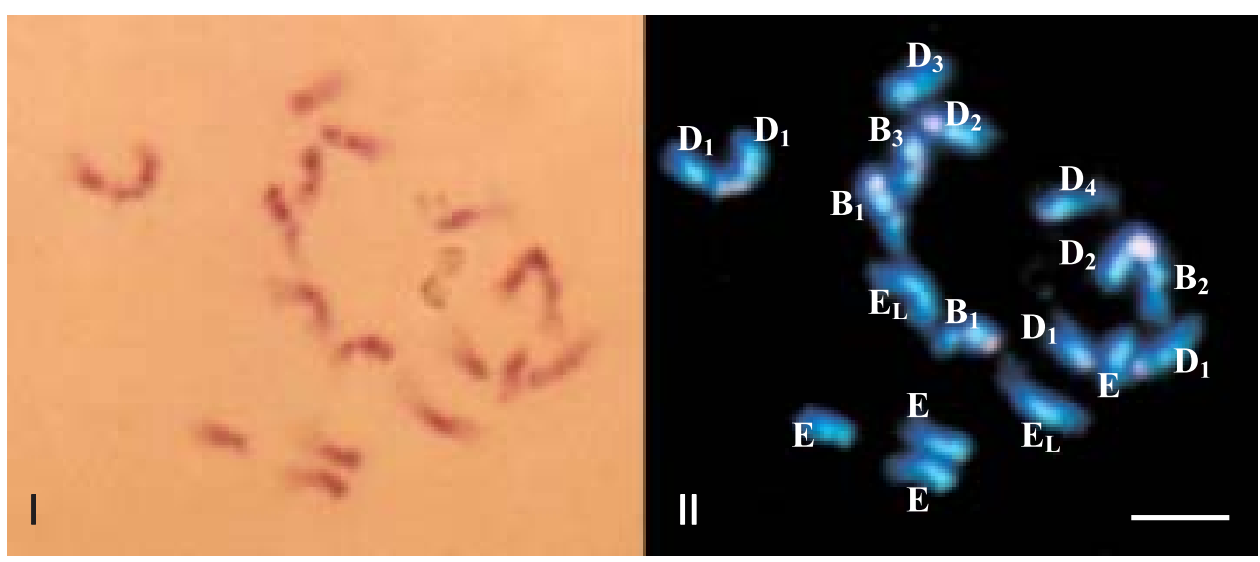

Fig. 2. Chromosomes of trifoliate orange stained with Giemsa (I) and PI/DAPI (II). The letters $B_{1}, B_{2}, B_{3}, D_{1}, D_{2}, D_{3}, D_{4}, E_{L}$, and $E$ in photograph II indicate chromosome types, respectively, $2 \mathrm{n}=18$. Scale bar indicates $5 \mu \mathrm{m}$.

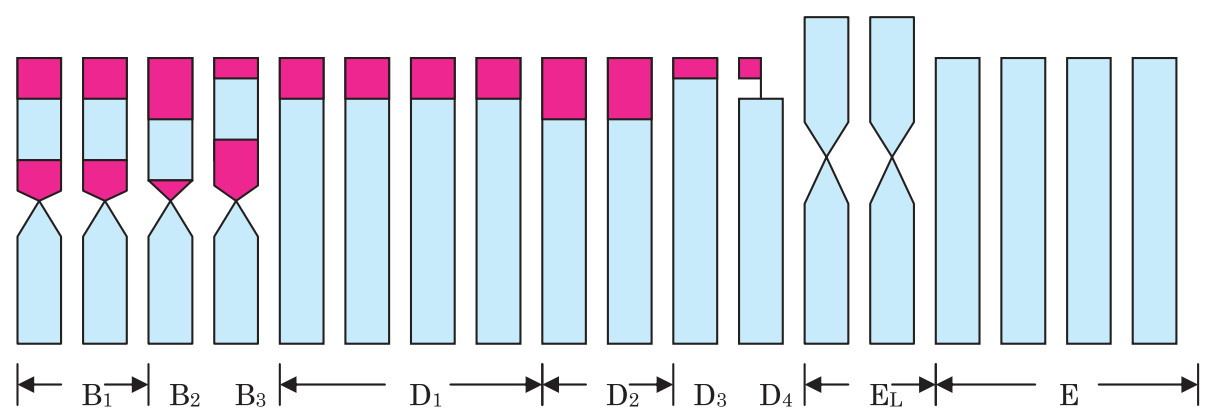

Fig. 3. Schematic representation of trifoliate orange chromosomes according to the position of PI-positive (PI $(+))$ band patterns. $B_{1}$-type chromosomes have a secondary constriction of the PI $(+)$ region separate from each other and a relatively medium intensity telomeric PI $(+)$ region. $\mathrm{B}_{2}$-type chromosomes have a small intensity secondary constriction of the PI $(+)$ region and large intensity telomeric PI $(+)$ region. $\mathrm{B}_{3}$-type chromosomes have a large intensity secondary constriction of the PI $(+)$ region and small intensity telomeric PI $(+)$ region. The $\mathrm{D}_{1}$-type chromosome showed an intermediate intensity telomeric $\mathrm{PI}(+)$ region. $\mathrm{D}_{2}$-type chromosomes have a large intensity telomeric PI (+) region. $\mathrm{D}_{3}$-type chromosomes have a small intensity telomeric PI $(+)$ region. $\mathrm{D}_{4}$-type chromosomes have a large intensity sharp morphology of the telomeric PI (+) region. $\mathrm{E}_{\mathrm{L}}$-type chromosomes have no PI (+) region, are relatively long, and the centromere is distinct. E-type chromosomes have no PI $(+)$ region, are of medium length, and the centromere is not distinct.

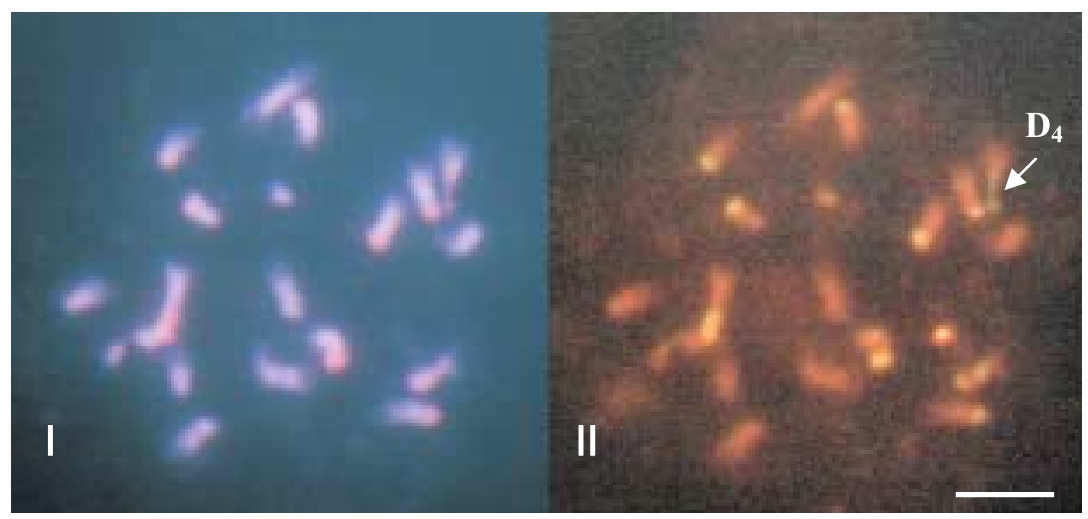

Fig. 4. Chromosomes of trifoliate orange stained with PI/DAPI (I) and genomic in situ hybridization (GISH) using satsuma mandarin DNA as a Bio-labeled probe (II). The arrow in photograph II indicates one green signal on the telomeric region of the $\mathrm{D}_{4}$ chromosome. Scale bar indicates $5 \mu \mathrm{m}$.

\section{GISH for chromosomes}

In GISH, the signals on trifoliate orange chromosomes revealed identical positions of CMA $(+)$ and PI $(+)$ regions with higher intensity. Sixteen signals (15 yellow $(\mathrm{Y})$ and 1 green $(\mathrm{G})$ ) were detected using a Bio-labeled satsuma mandarin DNA probe, as shown in Figures 4 and 5. One green signal was detected on $\mathrm{D}_{4}$ chromosomes. Trifoliate orange chromosomes were classified by colorization of the satsuma mandarin DNA probe into $2 \mathrm{~B}_{1}(\mathrm{Y}-\mathrm{Y})+1 \mathrm{~B}_{2}(\mathrm{Y}-\mathrm{Y})+1 \mathrm{~B}_{3}(\mathrm{Y}-\mathrm{Y})+$ 


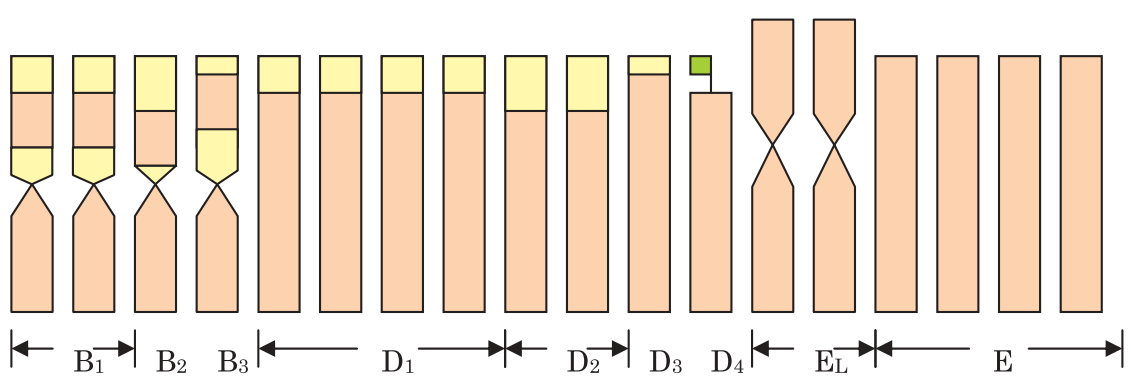

Fig. 5. Schematic representation of trifoliate orange chromosomes according to the position and the colorization of GISH using satsuma mandarin DNA as a Bio-labeled probe.

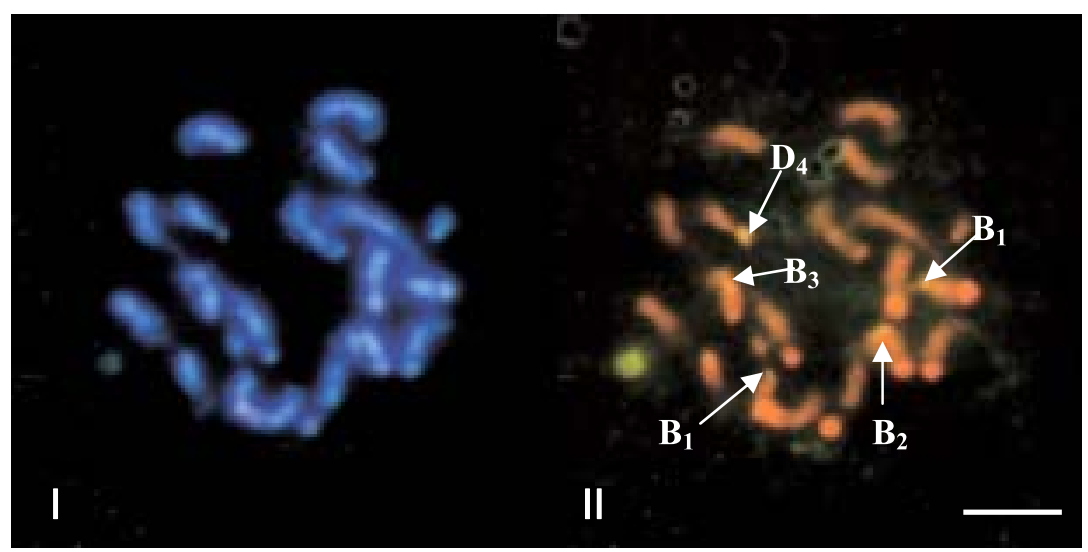

Fig. 6. Chromosomes of trifoliate orange stained with PI/DAPI (I) and GISH using 'Tosa Buntan' pummelo DNA as a Bio-labeled probe (II). The arrows in photograph II indicate 5 yellow signals, on the secondary constriction of the B-type chromosome and telomeric region of the $\mathrm{D}_{4}$-type chromosome. Scale bar indicates $5 \mu \mathrm{m}$.

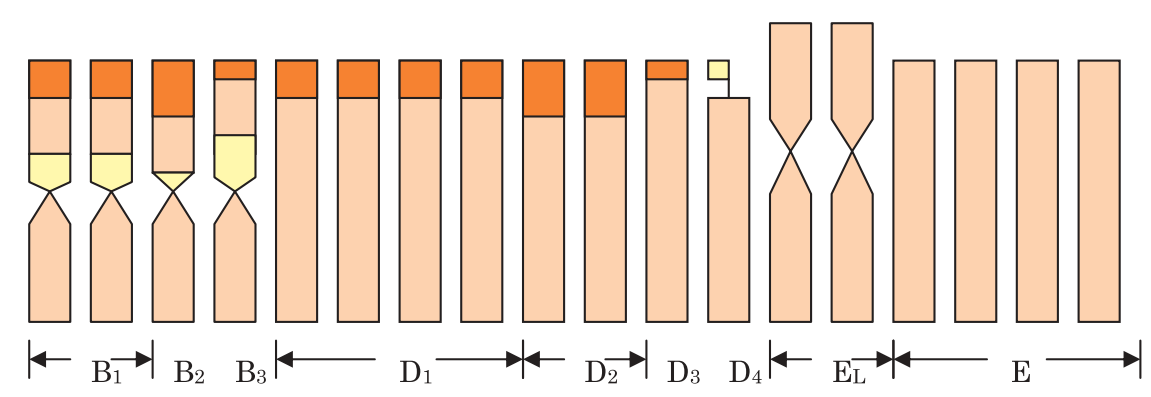

Fig. 7. Schematic representation of trifoliate orange chromosomes according to the position and colorization of GISH using 'Tosa Buntan' pummelo DNA as a Bio-labeled probe.

$4 \mathrm{D}_{1}(\mathrm{Y})+2 \mathrm{D}_{2}(\mathrm{Y})+1 \mathrm{D}_{3}(\mathrm{Y})+1 \mathrm{D}_{4}(\mathrm{G})+2 \mathrm{E}_{\mathrm{L}}+4 \mathrm{E} . \mathrm{In}$ the Bio-labeled 'Tosa Buntan' pummelo DNA probe, 11 red $(\mathrm{R})$ signals and 5 yellow signals were detected, as shown in Figures 6 and 7. Four yellow signals were detected on each secondary constriction of all B-type chromosomes and the other one yellow signal was detected on the $\mathrm{D}_{4}$ chromosome. Trifoliate orange chromosomes were classified by colorization of the 'Tosa Buntan' pummelo DNA probe into $2 \mathrm{~B}_{1}(\mathrm{R}-\mathrm{Y})+$ $1 \mathrm{~B}_{2}(\mathrm{R}-\mathrm{Y})+1 \mathrm{~B}_{3}(\mathrm{R}-\mathrm{Y})+4 \mathrm{D}_{1}(\mathrm{R})+2 \mathrm{D}_{2}(\mathrm{R})+1 \mathrm{D}_{3}(\mathrm{R})$ $+1 \mathrm{D}_{4}(\mathrm{Y})+2 \mathrm{E}_{\mathrm{L}}+4 \mathrm{E}$. Chromosomes in the same types of band patterns with relative sizes of signals could not be classified into more detailed categories by GISH signals of both satsuma mandarin and 'Tosa Buntan' pummelo DNA probes.

\section{Discussion}

CMA banding patterns of CMA $(+)$ regions in trifoliate orange chromosomes observed in the present study were identical to those previously reported by Befu et al. (2000). Based on this classification of chromosomes, trifoliate orange chromosomes were identified as $4 \mathrm{~B}+$ $8 \mathrm{D}+6 \mathrm{E}$. However, these results differ from those of Miranda et al. (1997a), who found different karyotyping patterns of trifoliate orange, $2 \mathrm{~B}+10 \mathrm{D}+6 \mathrm{E}$, using root tip samples. As Miranda et al. (1997a) selected samples from nucellar seedlings, there would be different types of trifoliate orange. Yoshida (1985) described wide 
variations in morphology and physiology, indicating that trifoliate oranges were heterozygous, possibly because of containing some strains derived from natural hybrids with Citrus, and that the morphological features of the hybrids usually resembled Poncirus. Moreover, Fang et al. (1997) revealed that the 48 trifoliate orange accessions could be classified into four major groups based on polymorphic ISSR markers. Thus, it may be difficult to determine a true Poncirus.

Although CMA staining made it possible to observe the banding patterns of positive signals in chromosomes, CMA signals were sometimes not strong enough to subclassify the chromosomes and easily faded during photographing by an epifluorescence microscope, as shown in Figure 1. The comparative analysis of CMA/ DAPI and PI/DAPI showed common positive bands of CMA (+) and PI (+) located at the same positions on chromosomes. PI/DAPI staining was a very simple procedure and revealed distinct chromosome types with enlarged sizes of PI $(+)$ regions and highly stable positive signals on a longer exposure time of the epifluorescence microscope. Therefore, PI/DAPI staining could be a superior choice to CMA/DAPI for chromosome karyotyping in citrus.

The karyotype generally shows common characteristics in size and band distribution patterns. However, the difference in size among citrus chromosomes was often very small and there is a possibility that chromosome order by size may change among cells. In addition, precise measurements of the lengths of positive CMA and PI regions were not easily performed. Befu et al. (2002) reported that the relative sizes of CMA $(+)$ signals were useful as an index for chromosome classification. In this study, therefore, the relative sizes of PI $(+)$ signals were used as an index to compare chromosomes for classification and to demonstrate chromosomes for the summarized schematic. The results in this study based on PI band patterns with relative sizes of PI $(+)$ signals revealed that all 18 chromosomes of trifoliate orange were classified into 9 groups, $2 \mathrm{~B}_{1}+1 \mathrm{~B}_{2}+1 \mathrm{~B}_{3}+4 \mathrm{D}_{1}+$ $2 \mathrm{D}_{2}+1 \mathrm{D}_{3}+1 \mathrm{D}_{4}+2 \mathrm{E}_{\mathrm{L}}+4 \mathrm{E}$, with a simple description and comparison. Thus, the relative sizes of PI $(+)$ signals were a very useful index for trifoliate orange chromosome identification.

Via GISH, signals on trifoliate orange chromosomes were detected at identical positions of CMA $(+)$ and PI $(+)$ regions. According to the previous measurement of CMA-positive regions (Miranda et al., 1997a), Citrus chromosomes have a large amount of heterochromatin. In the CMA/DAPI counterstaining, the CMA- and DAPIpositive signals on chromosomes corresponded to GCrich and AT-rich regions, respectively. The main type of $\mathrm{CMA}(+)$ was $\mathrm{CMA}^{+} / \mathrm{DAPI}^{-}$heterochromatin. In addition, stretched segments were frequently found and the segments would be represented as the secondary constriction which, as in many other species, was a deeply DAPI-negative (DAPI ${ }^{--}$) region (Carvalho et al.,
2005; Cornelio et al., 2003; Miranda et al., 1997a). On the other hand, PI generally conjugates to non-specific AT- and GC-rich DNA regions of chromosomes (Miranda et al., 1997b). However, in this study, CMA $(+)$, PI (+), and also GISH signals were detected at the same positions of the telomere and secondary constriction regions. The telomere and secondary constriction always refer to condensed chromosomal regions and a specific repeated nucleotide sequence, which are often GC-rich (Miller, 1981). Therefore, the regions in which detected CMA (+), PI (+), and GISH signals may contain highly condensed nucleotide sequences.

In the present study, since the trifoliate orange DNA labeled with Dig and conjugated to anti-Dig-rhodamine (red) was hybridized with trifoliate orange chromosomes as a probe, the other probe was detected using the Biolabeled DNA of satsuma mandarin or 'Tosa Buntan' pummelo conjugated to FITC (green) by GISH. The two probes of Dig- and Bio-labeled DNA are hybridized simultaneously and the color of the fluorescence was a mixed one from the two probes (generally yellow). If, however, a chromosomal segment is shared by common DNA sequences and the probe contains sufficient amounts, green fluorescence is seen on that segment after hybridization. Conversely, in the case of specific DNA sequences in chromosomal segments, the fluorescence of regions tends to be red after hybridization. The development of red, yellow, and green signals by GISH made it possible to classify chromosome types more clearly than via CMA and PI/DAPI staining. Especially, in $\mathrm{B}_{1}$ - and $\mathrm{B}_{2}$-type chromosomes, the signals on the secondary constriction were detected by GISH more easily, while those were sometimes very weak by CMA or PI staining. However, GISH using both satsuma mandarin and 'Tosa Buntan' pummelo biotin probes could not classify trifoliate orange chromosomes into more detailed categories than PI/DAPI staining. On the other hand, 15 yellow signals were detected by the satsuma mandarin probe, while 11 red signals were detected by the 'Tosa Buntan' pummelo probe. This suggests that the genomes of trifoliate orange and satsuma mandarin share many common DNA sequences, and trifoliate orange is more closely related to satsuma mandarin than 'Tosa Buntan' pummelo. Yamamoto and Kobayashi (1996) reported, based on RFLP analysis of chloroplast DNA, that the fragment patterns of citron, mandarin, Poncirus, and Fortunella were always identical, while those of pummelo were always identical with grapefruit, sour orange, and sweet orange. These results suggest that cytoplasmic genomes of mandarin and Poncirus from basic species are more closely related than pummelo, corresponding to the present study results.

In addition, signals on the secondary constriction of B-type chromosomes and the telomeric region of $\mathrm{D}_{4}$ type chromosomes were yellow or green with the Bio- 
labeled satsuma mandarin probe, and yellow with the Bio-labeled 'Tosa Buntan' pummelo probe. These result indicate that these regions have many shared DNA sequences, like conserved regions, even among distantly related species. Pedrosa et al. (2000) reported that three $45 \mathrm{~S}$ rDNA sites were comprised of CMA-positive regions in C. sinensis (L.) Osbeck: one in the terminal region of the D-type chromosome and one in the proximal region of both B-type chromosomes. Miranda et al. (1997b) reported that four rDNA sites corresponded to CMA-positive; in the centromeric region of both Atype chromosomes and in the telomeric region of both C-type chromosomes in Meiwa Kumquat (Fortunella crassifolia Swing.). These findings indicate that rDNA sites were comprised of CMA $(+)$ regions of the telomere in the C- and D-type, and of secondary constriction in the A- and B-type in citrus chromosomes. The secondary constrictions correspond to the nucleolar-organizing region (nucleolus organizer region, NOR), where the $45 \mathrm{~S}$ ribosomal RNA gene is tandemly clustered. Therefore, it is suggested that the secondary constriction of B-type chromosomes, in which yellow GISH signals were detected by both satsuma mandarin and 'Tosa Buntan' pummelo probes, contains tandemly clustered $45 \mathrm{~S}$ ribosomal RNA genes in trifoliate orange. In addition, the telomeric region of the $\mathrm{D}_{4}$-type chromosome in which green or yellow GISH signals were detected, is possibly related to rDNA sites in trifoliate orange. Moreover, it is suggested that these regions of $\mathrm{B}$ - and $\mathrm{D}_{4}$-type chromosomes in trifoliate orange can be homologous to satsuma mandarin and 'Tosa Buntan' pummelo. Befu et al. $(2000,2001)$ reported that CMA banding patterns in satsuma mandarin and 'Tosa Buntan' pummelo were $1 \mathrm{~A}+1 \mathrm{C}+8 \mathrm{D}+8 \mathrm{E}$ and $1 \mathrm{~A}+1 \mathrm{~B}+4 \mathrm{C}+$ $2 \mathrm{D}+9 \mathrm{E}$, respectively. B-type chromosomes do not exist in satsuma mandarin and $\mathrm{D}_{4}$-type chromosomes exist in neither satsuma mandarin nor 'Tosa Buntan' pummelo. It can be considered that the homologous regions in trifoliate orange are conserved on different types of chromosomes in satsuma mandarin and 'Tosa Buntan' pummelo. If this is true, this is important for Citrus and its closely related genera, as this would become a key to clarify the path of Citrus, Poncirus, and Fortunella chromosome evolution. It will become possible to identify some homologous chromosomes among Citrus, Poncirus, and Fortunella by GISH with different combinations of chromosome species/genera and probe species/genera.

\section{Literature Cited}

Befu, M., A. Kitajima and K. Hasegawa. 2001. Chromosome composition of some citrus species and cultivars based on the chromomycin $\mathrm{A}_{3}$ (CMA) banding patterns. J. Japan. Soc. Hort. Sci. 70: 83-88 (In Japanese with English abstract).

Befu, M., A. Kitajima and K. Hasegawa. 2002. Classification of the citrus chromosomes with same types of chromomycin A banding patterns. J. Japan. Soc. Hort. Sci. 71: 394-400 (In Japanese with English abstract).
Befu, M., A. Kitajima, X. L. Yang and K. Hasegawa. 2000. Classification of 'Tosa-Buntan' pummelo (Citrus grandis [L.] Osb.), 'Washington' navel orange (C. sinensis [L.] Osb.) and trifoliate orange (Poncirus trifoliata [L.] Raf.) chromosomes using young leaves. J. Japan. Soc. Hort. Sci. 69: 22-28.

Cameron, J. W. and H. B. Frost. 1968. Genetics, breeding and nucellar embryony in Citrus. p. 325-370. In: W. Reuther, L. D. Batchelor and H. J. Webber (eds.). The citrus industry. vol. II. Univ. California Press, Riverside.

Carvalho, R., W. S. Soares Filho, A. C. Brasileiro-Vidal and M. Guerra. 2005. The relationships among lemons, limes and citron: a chromosomal comparison. Cytogenet. Genome Res. 109: 276-282.

Choi, Y. A., R. Tao, K. Yonemori and A. Sugiura. 2003. Genomic in situ hybridization between persimmon (Diospyros kaki) and several wild species of Diospyros. J. Japan. Soc. Hort. Sci. 72: 385-388.

Coletta Filho, H. D., M. A. Machado, M. L. P. N. Targon, M. C. P. Q. D. G.. Moreira and J. Pompeu Jr. 1998. Analysis of the genetic diversity among mandarins (Citrus spp.) using RAPD markers. Euphytica 102: 133-139.

Cornelio, M. T. M. N., A. R. S. Figueiroa, K. G. B. Santos, R. Carvalho, W. S. Soares Filho and M. Guerra. 2003. Chromosomal relationships among cultivars of Citrus reticulata Blanco, its hybrids and related species. Plant Syst. Evol. 240: 149-161.

Doyle, J. J. and J. L. Doyle. 1987. A rapid DNA isolation procedure for small quantities of fresh leaf tissue. Phytochem. Bull. 19: $11-15$.

Fang, D. Q., M. L. Roose, R. R. Krueger and C. T. Federici. 1997. Fingerprinting trifoliate orange germ plasm accessions with isozymes, RFLPs, and inter-simple sequence repeat markers. Theor. Appl. Genet. 95: 211-219.

Fang, D. Q., R. R. Krueger and M. L. Roose. 1998. Phylogenetic relationships among selected Citrus germplasm accessions revealed by inter-simple sequence repeat (ISSR) markers. J. Amer. Soc. Sci. 123: 612-617.

Gmitter, F. G., S. Y. Xiao, S. Huang, X. L. Hu, S. M. Garnsey and Z. Deng. 1996. A localized linkage map of the citrus tristeza virus resistance gene region. Theor. Appl. Genet. 92: 688-695.

Guerra, M. 1985. Cytogenetics of Rutaceae. III. Heterochromatin patterns. Caryologia 38: 335-346.

Guerra, M. 1993. Cytogenetics of Rutaceae. V. High chromosomal variability in Citrus species revealed by CMA/DAPI staining. Heredity 71: 234-241.

Hizume, M. 1994. Allodiploid nature of Allium wakegi Araki revealed by genomic in situ hybridization and localization of 5S and 18S rDNAs. Jpn. J. Genet. 69: 407-415.

Kitajima, A., M. Befu, Y. Hidaka, T. Hotta and K. Hasegawa. 2001. A chromosome preparation method using young leaves of Citrus. J. Japan. Soc. Hort. Sci. 70: 191-194.

Krug, C. A. 1943. Chromosome numbers in the subfamily Aurantioideae with special reference to the genus Citrus. Bot. Gaz. 104: 602-611.

Liang, G. 1988. Studies on the Giemsa C-banding patterns of some Citrus and its related genera. Acta. Genet. Sin. 15: 409-415.

Miller, O. L. 1981. The nucleolus, chromosomes, and visualization of genetic activity. J. Cell Biol. 91: 15s-27s.

Miranda, M., F. Ikeda, T. Endo, T. Moriguchi and M. Omura. 1997a. Comparative analysis on the distribution of heterochromatin in Citrus, Poncirus and Fortunella chromosomes. Chromosome Res. 5: 86-92.

Miranda, M., F. Ikeda, T. Endo, T. Moriguchi and M. Omura. 1997b. rDNA sites and heterochromatin in Meiwa kumquat 
(Fortunella crassifolia Swing.) chromosomes revealed by FISH and CMA/DAPI staining. Caryologia 50: 333-340.

Pedrosa, A., D. Schweizer and M. Guerra. 2000. Cytological heterozygosity and the hybrid origin of sweet orange $[C$. sinensis (L.) Osbeck]. Theor. Appl. Genet. 100: 361-367.

Raina, S. N., Y. Mukai and M. Yamamoto. 1998. In situ hybridization identifies the diploid progenitor species of Coffea arabica (Rubiaceae). Theor. Appl. Genet. 97: 12041209.
Wei, W., Y. Cheng and Y. Duan. 1988. Studies on the evolution of Citrus based on karyotype and Giemsa C-banding pattern. Acta. Hort. Sin. 15: 223-228.

Yamamoto, M. and S. Kobayashi. 1996. Polymorphism of chloroplast DNA in citrus. J. Japan. Soc. Hort. Sci. 65: 291296.

Yoshida, T. 1985. Inheritance of susceptibility to citrus tristeza virus in trifoliate orange (Poncirus trifoliata Raf.). Bull. Fruit Tree Res. Stn. B12: 17-25 (In Japanese with English abstract). 